BACTERIAL PHYSIOLOGY

\title{
Unravelling membrane transport
}

Membrane transport proteins are crucial for maintaining a selective internal cellular environment. The largest subset of membrane transport proteins is the major facilitator superfamily (MFS), which convert electrochemical gradients into substrate concentration gradients, and transport ions, sugars, sugar-phosphates, drugs, amino acids and other hydrophilic solutes. In bacteria, MFS proteins are primarily responsible for nutrient uptake, although some act as drug-efflux pumps and are involved in the development of antibiotic resistance.

Despite the importance of these proteins, there has been little progress in the determination of their structure and mechanism. However, recent articles in Science now report the first high-resolution X-ray crystal structures of two MFS proteins from Escherichia coli: lactose permease (LacY), which catalyses the symport of $\beta$-galactosidase with $\mathrm{H}^{+}$; and the glycerol-3-phosphate transporter (GlpT), which catalyses the exchange of glycerol-3-phosphate with inorganic phosphate. In the two papers, Abramson et al. report the structure of $\mathrm{LacY}$ in complex with the lactose homologue $\beta$-D-galactopyranosyl-1thio- $\beta$-D-galactopyranoside (TDG) to a resolution of $3.5 \AA$, and Huang et al. present the uncomplexed GlpT structure to 3.3-Å resolution.

Both LacY and GlpT are shaped like 'Mayan temples' and consist of two similar domains - the aminoand carboxy-teminal domains - each of which contains six transmembrane helices. A central hydrophilic cavity that is open to the cytoplasm, yet closed to the periplasm, is also present in both structures, giving the overall structure a 'heart-shaped' topology when viewed normal to the membrane. The TDG molecule in the LacY structure is bound in this hydrophilic cavity, and is positioned in the centre of the membrane, almost equidistant from both the cytoplasm and periplasm - consistent with the notion that MFS proteins have a single substrate-binding site. The highresolution structures have identified several residues important for substrate binding.

To allow transport of substrates across the membrane there must also be a structural conformation in which the cavity is open to the periplasm. Based on the crystal structures, the authors of each paper propose a transport mechanism for MFS proteins, in which protonation and binding of substrates induce several conformational changes. These conformational changes are postulated to result in 'switching' of the opening of the hydrophilic cavity from the periplasm (outward-facing) to the cytoplasm (inward-facing), thereby allowing transport of substrates across the membrane.

The authors have identified several residues from each structure as important in facilitating the conformational changes necessary for the transition between the outward- and inward-facing conformations, and if a structure of the outward-facing conformation could be obtained, this mechanism, and the MFS story, would be complete.

\section{Jane Saunders \\ (Q) References and links ORIGINAL RESEARCH PAPERS Abramson, J. et al. Structure and mechanism of the lactose permease of Escherichia coli. Science 301 610-615 (2003). \\ Huang, Y. et al. Structure and mechanism of the glycerol-3-phosphate transporter from Escherichia} coli. Science 301, 616-620 (2003).

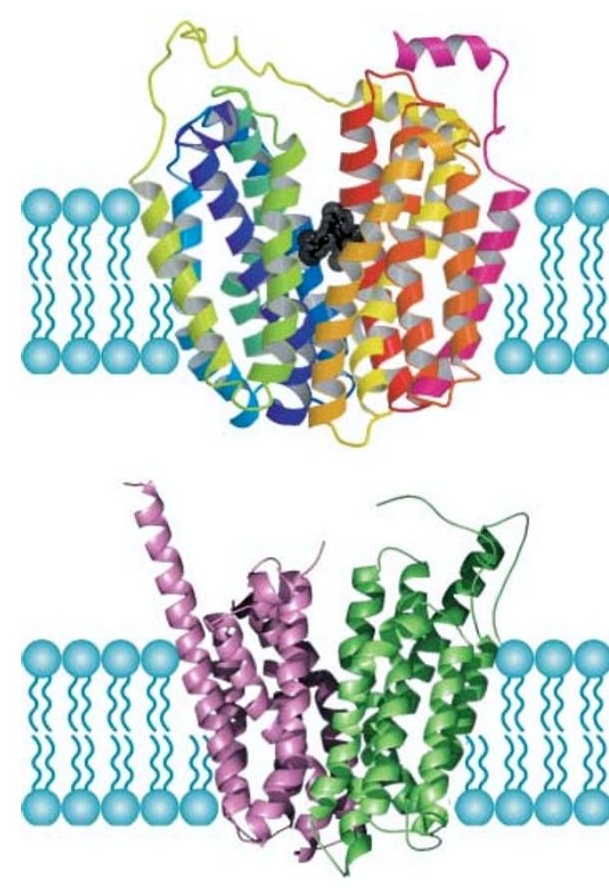

Crystal structures of LacY and GlpT are shown in the upper and lower panels, respectively. Images courtesy of Professor So Iwata (Imperial College, London, UK) and Professor Da-Neng Wang (New York University School of Medicine, USA). 